\title{
THE EMPIRICAL DISTRIBUTION FUNCTION FOR DEPENDENT VARIABLES: ASYMPTOTIC AND NONASYMPTOTIC RESULTS IN $\mathbb{L}^{p}$
}

\author{
JÉrôme Dedecker ${ }^{1}$ And Florence MERlevède ${ }^{2}$
}

\begin{abstract}
Considering the centered empirical distribution function $F_{n}-F$ as a variable in $\mathbb{L}^{p}(\mu)$, we derive non asymptotic upper bounds for the deviation of the $\mathbb{L}^{p}(\mu)$-norms of $F_{n}-F$ as well as central limit theorems for the empirical process indexed by the elements of generalized Sobolev balls. These results are valid for a large class of dependent sequences, including non-mixing processes and some dynamical systems.
\end{abstract}

Mathematics Subject Classification. 60F10, 62G30.

Received September 20, 2005. Revised May 22, 2006.

\section{INTRODUCTION}

Let $\left(X_{i}\right)_{1 \leq i \leq n}$ be equidistributed real-valued random variables with common distribution function $F$. Let $F_{n}$ be the empirical distribution function $F_{n}(t)=n^{-1} \sum_{i=1}^{n} \mathbf{I}_{X_{i} \leq t}$. Let $1 \leq p<\infty$ and $\mu$ be a $\sigma$-finite measure on $\mathbb{R}$. Suppose that $F$ satisfies

$$
\int_{\mathbb{R}_{-}}(F(t))^{p} \mu(\mathrm{d} t)+\int_{\mathbb{R}_{+}}(1-F(t))^{p} \mu(\mathrm{d} t)<\infty .
$$

Under this assumption, the process $\left\{t \rightarrow F_{n}(t)-F(t), t \in \mathbb{R}\right\}$ may be viewed as a random variable with values in the space $\mathbb{L}^{p}(\mu)$. Let $\|\cdot\|_{p, \mu}$ be the $\mathbb{L}^{p}$-norm with respect to $\mu$, and define

$$
D_{p, n}(\mu)=\left(\int\left|F_{n}(t)-F(t)\right|^{p} \mu(\mathrm{d} t)\right)^{1 / p}=\left\|F_{n}-F\right\|_{p, \mu} .
$$

When $p=2$ and $\mu=\mathrm{d} F, D_{2, n}^{2}(\mu)$ is known as the Cramér-von Mises statistics, and is commonly used for testing goodness of fit. When $p=1$ and $\lambda$ is the Lebesgue measure on the real line, then $D_{1, n}(\lambda)$ is the $\mathbb{L}^{1}(\lambda)$-minimal distance between $F_{n}$ and $F$, denoted in what follows by $K\left(F_{n}, F\right)$.

It is interesting to write $D_{p, n}(\mu)$ as the supremum of the empirical process over a particular class of functions. The proof of the following lemma is given in the appendix.

Keywords and phrases. Deviation inequalities, weak dependence, Cramér-von Mises statistics, empirical process, expanding maps.

${ }^{1}$ Laboratoire de Statistique Théorique et Appliquée, Université Paris 6, 175 rue du Chevaleret, 75013 Paris, France. dedecker@ccr.jussieu.fr

${ }^{2}$ Laboratoire de probabilités et modèles aléatoires, UMR 7599, Université Paris 6, 175 rue du Chevaleret, 75013 Paris, France. merleve@ccr.jussieu.fr 
Lemma 1. Let $F$ and $G$ be two distributions functions satisfying (1.1). If $p^{\prime}$ is the conjugate exponent of $p$, let $W_{p^{\prime}, 1}(\mu)$ be the set of functions

$$
\left\{f: f(t)=f(0)+\left(\int_{[0, t[} f^{\prime}(x) \mu(\mathrm{d} x)\right) \mathbf{I}_{t>0}-\left(\int_{[t, 0[} f^{\prime}(x) \mu(\mathrm{d} x)\right) \mathbb{I}_{t \leq 0},\left\|f^{\prime}\right\|_{p^{\prime}, \mu} \leq 1\right\} .
$$

For any $f$ in $W_{p^{\prime}, 1}(\mu)$, we have

$$
\int f \mathrm{~d} F-\int f \mathrm{~d} G=\mu\left(f^{\prime}(F-G)\right) \quad \text { so that } \quad\|F-G\|_{p, \mu}=\sup _{f \in W_{p^{\prime}, 1}(\mu)}\left|\int f \mathrm{~d} F-\int f \mathrm{~d} G\right| .
$$

According to Lemma 1 , since $D_{p, n}(\mu)=\left\|F_{n}-F\right\|_{p, \mu}$, we have that

$$
D_{p, n}(\mu)=\sup _{f \in W_{p^{\prime}, 1}(\mu)}\left|\frac{1}{n} \sum_{i=1}^{n} f\left(X_{i}\right)-\mathbb{E}\left(f\left(X_{i}\right)\right)\right|
$$

If $\lambda_{I}$ is the Lebesgue measure on the interval $I \subset \mathbb{R}, W_{p^{\prime}, 1}\left(\lambda_{I}\right)$ is the space of absolutely continuous functions $f$ such that $\lambda_{I}\left(\left|f^{\prime}\right| p^{\prime}\right) \leq 1$ (hence it contains the unit ball of the Sobolev space of order 1 with respect to $\mathbb{L}^{p^{\prime}}\left(\lambda_{I}\right)$ ). Let us now recall what is known about the entropy properties of the class: $W_{p^{\prime}, 1}^{0}\left(\lambda_{I}\right)=\left\{f-f(0), f \in W_{p^{\prime}, 1}\left(\lambda_{I}\right)\right\}$. If $I$ is compact and if $1<p^{\prime} \leq \infty$ (or equivalently $1 \leq p<\infty$ ), then, according to Corollary 1 in Birgé and Massart (2000) [3], the space $W_{p^{\prime}, 1}^{0}\left(\lambda_{I}\right)$ is compact with respect to the $\mathbb{L}^{\infty}$-norm with $\varepsilon$-entropy of order $\varepsilon^{-1}$. Of course this is no longer true if $I$ is not compact, and as far as we know, the entropy properties of these classes have not been studied. However, arguing as in van der Vaart (1994) [17], one can prove that the $\varepsilon-\mathbb{L}^{r}(P)$ bracketing entropy with respect to a probability $P$ is of order $\varepsilon^{-1}$ provided $\sum_{n \in \mathbb{Z}}\left(|n|^{r} P([n, n+1])\right)^{1 /(1+r)}<\infty$. To our knowledge, no entropy bounds are available for the class $W_{p^{\prime}, 1}^{0}(\mu)$ when $\mu$ is not the Lebesgue measure on some interval.

These entropy bounds can be used to prove uniform central limit theorems and maximal inequalities for the empirical process indexed by elements of $W_{p^{\prime}, 1}^{0}\left(\lambda_{I}\right)$ in the iid case (see again van der Vaart (1994) [17], Sect. 4). Some of these results can be extended to the dependent context, but the general results based on entropy methods are only available in a mixing context (see Rem. 6 below). Our aim in this paper is to show that we can obtain asymptotic and non-asymptotic results for the empirical process indexed by elements of $W_{p^{\prime}, 1}(\mu)$, for some dependence coefficients which are perfectly adapted to the class $W_{p^{\prime}, 1}(\mu)$.

In Section 2, we give a nonasymptotic upper bound for the deviation of $D_{p, n}(\mu)$ when $2 \leq p<\infty$. In Section 3 we study the weak convergence of $\sqrt{n}\left(F_{n}-F\right)$ in the spaces $\mathbb{L}^{p}(\mu)$ when $2 \leq p<\infty$, which in turn is equivalent to the weak convergence of the normalized empirical process indexed by elements of the class $W_{p^{\prime}, 1}(\mu)$ (see Lems. 2 and 3). In both cases, the conditions are expressed in terms of some natural dependence coefficients, which can be viewed as mixing coefficients restricted to the class $W_{p^{\prime}, 1}(\mu)$ (see Def. 1 below). In Section 4, we compare these coefficients to other well known measures of dependence, and we show how they can be computed for two large classes of examples, including many non-mixing processes. In Section 5, we apply our results to the case of iterates of expanding maps of the unit interval, and to the simple example of linear processes.

\section{EXPONENTIAL BOUNDS}

We first define the dependence coefficients which naturally appear in this context.

Definition 1. Let $(\Omega, \mathcal{A}, \mathbb{P})$ be a probability space, let $X$ be a real-valued random variable and let $\mathcal{M}$ be a $\sigma$-algebra of $\mathcal{A}$. Let $\|\cdot\|_{p}$ be the the $\mathbb{L}^{p}$-norm with respect to $\mathbb{P}$. Denote by $\mathbb{P}_{X}$ the distribution of $X$ and by 
$\mathbb{P}_{X \mid \mathcal{M}}$ a regular distribution of $X$ given $\mathcal{M}$. Let $\left.\left.F_{X}(t)=\mathbb{P}_{X}(]-\infty, t\right]\right)$ and $\left.\left.F_{X \mid \mathcal{M}}(t)=\mathbb{P}_{X \mid \mathcal{M}}(]-\infty, t\right]\right)$. For $1 \leq p \leq \infty$ and $1 \leq q \leq \infty$, define the coefficient

$$
\tau_{\mu, p, q}(\mathcal{M}, X)=\left\|\left(\int\left|F_{X \mid \mathcal{M}}(t)-F_{X}(t)\right|^{p} \mu(\mathrm{d} t)\right)^{1 / p}\right\|_{q}=\|\| F_{X \mid \mathcal{M}}-F_{X}\left\|_{p, \mu}\right\|_{q} .
$$

From Lemma 1, we see that an equivalent definition is

$$
\tau_{\mu, p, q}(\mathcal{M}, X)=\left\|\sup _{f \in W_{p^{\prime}, 1}(\mu)}\left|\int f \mathrm{~d} F_{X \mid \mathcal{M}}-\int f \mathrm{~d} F_{X}\right|\right\|_{q}
$$

Theorem 1. Let $(\Omega, \mathcal{A}, \mathbb{P})$ be a probability space, and $\left(X_{i}\right)_{1 \leq i \leq n}$ be equidistributed real-valued random variables with common distribution function $F$. Let $\mathcal{M}_{0}=\{\emptyset, \Omega\}$ and let $\left(\mathcal{M}_{k}\right)_{1 \leq k \leq n}$ be an increasing sequence of $\sigma$ algebra such that $\sigma\left(X_{i}, 1 \leq i \leq k\right) \subseteq \mathcal{M}_{k}$. For any $2 \leq p<\infty$, any finite measure $\mu$ and any positive $x$, we have the upper bound

$$
\mathbb{P}\left(\sqrt{n} D_{p, n}(\mu) \geq x\right) \leq 2 \exp \left(-\frac{n x^{2}}{2(p-1) \sum_{i=1}^{n}\left(\sum_{k=i}^{n}\|\| F_{X_{k} \mid \mathcal{M}_{i}}-F_{X_{k} \mid \mathcal{M}_{i-1}}\left\|_{p, \mu}\right\|_{\infty}\right)^{2}}\right) .
$$

In particular, if

we have the upper bound

$$
C(p, n, \mu)=\sum_{i=1}^{n}\left(\sum_{k=i}^{n}\left(\tau_{\mu, p, \infty}\left(\mathcal{M}_{i}, X_{k}\right)+\tau_{\mu, p, \infty}\left(\mathcal{M}_{i-1}, X_{k}\right)\right)\right)^{2}
$$

$$
\mathbb{P}\left(\sqrt{n} D_{p, n}(\mu) \geq x\right) \leq 2 \exp \left(-\frac{n x^{2}}{2(p-1) C(p, n, \mu)}\right) .
$$

Remark 1. Let $\tau_{\mu, p, q}(i)=\max _{1 \leq k \leq n-i} \tau_{\mu, p, q}\left(\mathcal{M}_{k}, X_{k+i}\right)$ and $Z_{i}=\left\{t \rightarrow \mathbb{I}_{X_{i} \leq t}-F(t)\right\}$. Since

$$
\begin{aligned}
C(p, n, \mu) & \leq \sum_{i=1}^{n}\left(\|\| Z_{1}\left\|_{p, \mu}\right\|_{\infty}+\sum_{k=1}^{n-i} \tau_{\mu, p, \infty}(k)+\left(\sum_{k=1}^{n-i+1} \tau_{\mu, p, \infty}(k)\right) \mathbf{I}_{i>1}\right)^{2} \\
& \leq n\left(\|\| Z_{1}\left\|_{p, \mu}\right\|_{\infty}+2 \sum_{k=1}^{n-1} \tau_{\mu, p, \infty}(k)\right)^{2}
\end{aligned}
$$

we obtain from $(2.2)$ that

$$
\mathbb{P}\left(\sqrt{n} D_{p, n}(\mu) \geq x\right) \leq 2 \exp \left(-\frac{x^{2}}{2(p-1)\left(\|\| Z_{1}\left\|_{p, \mu}\right\|_{\infty}+2 \sum_{k=1}^{n-1} \tau_{\mu, p, \infty}(k)\right)^{2}}\right) .
$$

Proof of Theorem 1. Let $Z_{i}$ be as in Remark 1 and $S_{n}=\sum_{i=1}^{n} Z_{i}$. Clearly, we get that

$$
\sqrt{n} D_{p, n}(\mu)=\frac{\left\|S_{n}\right\|_{p, \mu}}{\sqrt{n}}
$$

We apply the method of martingale differences, as done in Yurinskii (1974) [19]. Since $\mathbb{E}\left(S_{n} \mid \mathcal{M}_{0}\right)=\mathbb{E}\left(S_{n}\right)=0$, we have that $S_{n}=\sum_{i=1}^{n}\left(\mathbb{E}\left(S_{n} \mid \mathcal{M}_{i}\right)-\mathbb{E}\left(S_{n} \mid \mathcal{M}_{i-1}\right)\right)$. For all $1 \leq i \leq n$, let $d_{i, n}=\mathbb{E}\left(S_{n} \mid \mathcal{M}_{i}\right)-\mathbb{E}\left(S_{n} \mid \mathcal{M}_{i-1}\right)$. 
Clearly $d_{i, n}$ is an $\mathcal{M}_{i}$-measurable random variable with values in $\mathbb{L}^{p}(\mu)$ such that $\mathbb{E}\left(d_{i, n} \mid \mathcal{M}_{i-1}\right)=0$ almost surely. From (2.4) and Theorem 3 in Pinelis (1992) [15], we infer that for any positive real $x$,

$$
\mathbb{P}\left(\sqrt{n} D_{p, n}(\mu) \geq x\right) \leq 2 \exp \left(-\frac{n x^{2}}{2(p-1) b_{n}^{2}}\right), \quad \text { where } b_{n}^{2}=\sum_{i=1}^{n}\|\| d_{i, n}\left\|_{p, \mu}\right\|_{\infty}^{2}
$$

The inequality (2.1) follows by noting that

$$
d_{i, n}=\sum_{k=i}^{n} F_{X_{k} \mid \mathcal{M}_{i}}-F_{X_{k} \mid \mathcal{M}_{i-1}}
$$

The inequality (2.2) follows from (2.1) by noting that

\|\|$F_{X_{k} \mid \mathcal{M}_{i}}-F_{X_{k} \mid \mathcal{M}_{i-1}}\left\|_{p, \mu}\right\|_{\infty} \leq \tau_{\mu, p, \infty}\left(\mathcal{M}_{i}, X_{k}\right)+\tau_{\mu, p, \infty}\left(\mathcal{M}_{i-1}, X_{k}\right)$

Remark 2. The bounds of Theorem 1 are valid for $2 \leq p<\infty$. If $p$ belongs to $\left[1,2\left[\right.\right.$, the space $\mathbb{L}^{p}(\mu)$ is no longer smooth, so that the method of martingale differences in Banach spaces does not work. Hence a reasonable question is: does (2.2) still holds (with possibly different constants) if $p$ belongs to $[1,2[$ ? This would give a bound in terms of the coefficients $\tau_{\mu, 1, \infty}$ which are the weakest among the coefficients $\tau_{\mu, p, \infty}$.

Of course, since $D_{p, n}(\mu) \leq D_{2, n}(\mu)$ for any probability $\mu$ and any $1 \leq p \leq 2$, Theorem 1 provides an upper bound for the deviation of $D_{p, n}(\mu)$ in terms of the coefficient $\tau_{\mu, 2, \infty}$ (and hence in terms of $\tau_{\mu, 1, \infty}$ since $\left.\tau_{\mu, 2, \infty}(\mathcal{M}, X) \leq\left(\tau_{\mu, 1, \infty}(\mathcal{M}, X)\right)^{1 / 2}\right)$. If the $X_{i}^{\prime}$ s are in $[0,1]$ and $\lambda_{1}$ is the Lebesgue measure on [0,1], we obtain a bound for the deviation of the Kantorovitch distance $K\left(F_{n}, F\right)=\left\|F_{n}-F\right\|_{1, \lambda_{1}}$ : for any positive $x$,

$$
\mathbb{P}\left(\sqrt{n} K\left(F_{n}, F\right) \geq x\right) \leq \mathbb{P}\left(\sqrt{n} D_{2, n}\left(\lambda_{1}\right) \geq x\right) \leq 2 \exp \left(-\frac{n x^{2}}{2 C\left(2, n, \lambda_{1}\right)}\right)
$$

From Remark 1, we also have, for a sequence $\left(X_{i}\right)_{i \geq 1}$ of variables with values in $[0,1]$,

$$
\mathbb{P}\left(\sqrt{n} K\left(F_{n}, F\right) \geq x\right) \leq \mathbb{P}\left(\sqrt{n} D_{2, n}\left(\lambda_{1}\right) \geq x\right) \leq 2 \exp \left(-\frac{x^{2}}{2\left(\|\| Z_{1}\left\|_{2, \lambda_{1}}\right\|_{\infty}+2 \sum_{k=1}^{n-1} \tau_{\lambda_{1}, 2, \infty}(k)\right)^{2}}\right)
$$

Remark 3. If $\mu=\delta_{t}$, we are looking for the deviation of $\sqrt{n}\left|F_{n}(t)-F(t)\right|$. Starting from (2.6), we see that $d_{i, n}(t)$ belongs to the interval $\left[A_{i}, B_{i}\right]$, where $A_{i}$ and $B_{i}$ are the $\mathcal{M}_{i-1}$-measurable random variables

$$
\begin{aligned}
A_{i} & =-F(t)-\sum_{k=i+1}^{n}\left\|\mathbb{E}\left(Z_{k}(t) \mid \mathcal{M}_{i}\right)\right\|_{\infty}-\sum_{k=i}^{n} \mathbb{E}\left(Z_{k}(t) \mid \mathcal{M}_{i-1}\right) \\
B_{i} & =(1-F(t))+\sum_{k=i+1}^{n}\left\|\mathbb{E}\left(Z_{k}(t) \mid \mathcal{M}_{i}\right)\right\|_{\infty}-\sum_{k=i}^{n} \mathbb{E}\left(Z_{k}(t) \mid \mathcal{M}_{i-1}\right) .
\end{aligned}
$$

The sum of the lengths $\left(B_{i}-A_{i}\right)^{2}$ is then $L(t, n)=\sum_{i=1}^{n}\left(1+2 \sum_{k=i+1}^{n}\left\|\mathbb{E}\left(Z_{k}(t) \mid \mathcal{M}_{i}\right)\right\|_{\infty}\right)^{2}$. Applying Azuma's inequality (1967) [1], we obtain

$$
\mathbb{P}\left(\sqrt{n}\left|F_{n}(t)-F(t)\right|>x\right) \leq 2 \exp \left(-\frac{2 n x^{2}}{L(t, n)}\right)
$$


Note that, for $\mu=\delta_{t},\left\|\mathbb{E}\left(Z_{k}(t) \mid \mathcal{M}_{i}\right)\right\|_{\infty}=\tau_{\mu, p, \infty}\left(\mathcal{M}_{i}, X_{k}\right)$, for any $1 \leq p \leq \infty$. For this choice of $\mu$, the bound (2.9) is much more precise than (2.2). In view of (2.9) one can wonder if (2.2) holds for any probability $\mu$ with

$$
L(p, n, \mu)=\sum_{i=1}^{n}\left(1+2 \sum_{k=i+1}^{n} \tau_{\mu, p, \infty}\left(X_{k}, \mathcal{M}_{i}\right)\right)^{2}
$$

instead of $4(p-1) C(p, n, \mu)$. We have no idea of how to prove such a bound. It is probably delicate, for if it is true then $\mathbb{P}\left(\sqrt{n} D_{p, n}(\mathrm{~d} F) \geq x\right) \leq 2 \exp \left(-2 x^{2}\right)$ for iid variables. If this bound holds for any $2 \leq p<\infty$, then it must hold for $p=\infty$, which is Massart's bound (1990) [11] for the deviation of Kolmogorov-Smirnov statistics.

\section{Empirical process indeXed in Sobolev Balls}

Let $(\Omega, \mathcal{A}, \mathbb{P})$ be a probability space and $T: \Omega \mapsto \Omega$ be a bijective bimeasurable transformation preserving the probability $\mathbb{P}$. An element $A$ of $\mathcal{A}$ is said to be invariant if $T(A)=A$. We denote by $\mathcal{I}$ the $\sigma$-algebra of all invariant sets.

Let $\mathcal{M}_{0}$ be a $\sigma$-algebra of $\mathcal{A}$ satisfying $\mathcal{M}_{0} \subseteq T^{-1}\left(\mathcal{M}_{0}\right)$ and define the nondecreasing filtration $\left(\mathcal{M}_{i}\right)_{i \in \mathbb{Z}}$ by $\mathcal{M}_{i}=T^{-i}\left(\mathcal{M}_{0}\right)$. Let $X_{0}$ be an $\mathcal{M}_{0}$-measurable real-valued random variable and define the sequence $\left(X_{i}\right)_{i \in \mathbb{Z}}$ by $X_{i}=X_{0} \circ T^{i}$. Define the coefficient $\tau_{\mu, p, q}(i)$ of the sequence $\left(X_{i}\right)_{i \in \mathbb{Z}}$ by

$$
\tau_{\mu, p, q}(i)=\tau_{\mu, p, q}\left(\mathcal{M}_{0}, X_{i}\right)
$$

Let $F$ be the distribution function of $X_{0}$ and $F_{n}$ be the empirical distribution function. Let $G_{n}$ be the centered and normalized empirical measure $G_{n}=\sqrt{n}\left(\mathrm{~d} F_{n}-\mathrm{d} F\right)$, and $\mathcal{F}$ be a class of measurable functions from $\mathbb{R}$ to $\mathbb{R}$. The space $\ell^{\infty}(\mathcal{F})$ is the space of all functions $z$ from $\mathcal{F}$ to $\mathbb{R}$ such that $\|z\|_{\mathcal{F}}=\sup _{f \in \mathcal{F}}|z(f)|$ is finite.

Let $1 \leq p<\infty$, let $p^{\prime}$ be the conjugate exponent of $p$, and let $\mu$ be any $\sigma$-finite measure such that (1.1) holds. We shall see that the convergence in distribution of $G_{n}$ indexed by the elements of $W_{p^{\prime}, 1}(\mu)$ is equivalent to the convergence in distribution of $\sqrt{n}\left(F_{n}-F\right)$ in $\mathbb{L}^{p}(\mu)$. We first define an appropriate isometry between $\mathbb{L}^{p}(\mu)$ and a subspace of $\ell^{\infty}\left(W_{p^{\prime}, 1}(\mu)\right)$.

Lemma 2. Given $f$ in $W_{p^{\prime}, 1}(\mu)$, let $f^{\prime}$ be as in Lemma 1. Let $h: \mathbb{L}^{p}(\mu) \mapsto \ell^{\infty}\left(W_{p^{\prime}, 1}(\mu)\right)$ be such that $h(g)=\left\{\mu\left(f^{\prime} g\right), f \in W_{p^{\prime}, 1}(\mu)\right\}$. The function $h$ is an isometry from $\mathbb{L}^{p}(\mu)$ to $h\left(W_{p^{\prime}, 1}(\mu)\right)=G_{p^{\prime}}(\mu)$. In particular, $G_{p^{\prime}}(\mu)$ is a separable Banach space.

Proof of Lemma 2. By duality $\left\|h\left(g_{1}\right)-h\left(g_{2}\right)\right\|_{W_{p^{\prime}, 1}(\mu)}=\left\|g_{1}-g_{2}\right\|_{p, \mu}$.

Now, by Lemma 1, $\left\{G_{n}(f), f \in W_{p^{\prime}, 1}(\mu)\right\}=h\left(\sqrt{n}\left(F_{n}-F\right)\right)$. Consequently, under (1.1), the empirical process $G_{n}$ indexed by the elements of $W_{p^{\prime}, 1}(\mu)$ is a random variable with values in $G_{p^{\prime}}(\mu)$. In addition, if $\gamma: G_{p^{\prime}}(\mu) \mapsto \mathbb{R}$ is continuous, then $\gamma \circ h: \mathbb{L}^{p}(\mu) \mapsto \mathbb{R}$ is also continuous. It follows immediately that

Lemma 3. If (1.1) holds, then the sequence $\left\{G_{n}(f), f \in W_{p^{\prime}, 1}(\mu)\right\}$ converges in distribution in the space $G_{p^{\prime}}(\mu)$ if and only if the sequence $\sqrt{n}\left(F_{n}-F\right)$ converges in distribution in the space $\mathbb{L}^{p}(\mu)$.

Consequently, central limit theorems for the empirical process indexed by the elements of $W_{p^{\prime}, 1}(\mu)$ can be deduced from central limit theorems for Banach-valued random variables. This approach leads to Theorem 2 below.

Theorem 2. Define the function $F_{\mu}$ by: $F_{\mu}(x)=\mu\left(\left[0, x[)\right.\right.$ if $x \geq 0$ and $F_{\mu}(x)=-\mu([x, 0[)$ if $x \leq 0$. Define also the nonnegative random variable $Y_{p, \mu}=\left|F_{\mu}\left(X_{0}\right)\right|^{1 / p}$ and assume that $\left\|Y_{p, \mu}\right\|_{2}<\infty$. Consider the three conditions

1. $p$ belongs to $\left[2, \infty\left[\right.\right.$, and $\sum_{k>0} \tau_{\mu, p, 2}(k)<\infty$.

2. $p=2, \mu(\mathbb{R})<\infty$, and $\sum_{k>0}^{k>0} \tau_{\mu, 2,1}(k)<\infty$. 
3. $p=2, F_{X_{0} \mid \mathcal{M}_{-\infty}}=F$, and $\sum_{k>0}\|\| F_{X_{k} \mid \mathcal{M}_{0}}-F_{X_{k} \mid \mathcal{M}_{-1}}\left\|_{2, \mu}\right\|_{2}<\infty$.

If one of these conditions holds, then the sequence $\left\{G_{n}(f), f \in W_{p^{\prime}, 1}(\mu)\right\}$ converges in distribution in the space $G_{p^{\prime}}(\mu)$ to a random variable whose conditional distribution with respect to $\mathcal{I}$ is that of a zero-mean Gaussian process with covariance function

$$
\Gamma(f, g)=\sum_{k \in \mathbb{Z}} \operatorname{Cov}\left(f\left(X_{0}\right), g\left(X_{k}\right) \mid \mathcal{I}\right)
$$

Remark 4. For $p=2,1$ implies 3. Note also that, if $\mu(\mathbb{R})<\infty$, then $Y_{p, \mu}$ is bounded by $\mu(\mathbb{R})^{1 / p}$.

Remark 5. According to Remark 6 in Dedecker and Merlevède (2003) [5], by noting that \|\| $\mathbb{E}\left(Z_{k} \mid \mathcal{M}_{0}\right)\left\|_{2, \mu}\right\|_{2}=$ $\tau_{\mu, 2,2}(k)$ with $Z_{k}$ defined in Remark 1 , the condition 3 is realized if there exists a sequence $\left(L_{k}\right)_{k>0}$ of positive numbers such that

$$
\sum_{i>0}\left(\sum_{k=1}^{i} L_{k}\right)^{-1}<\infty \text { and } \sum_{k>0} L_{k} \tau_{\mu, 2,2}^{2}(k)<\infty
$$

In particular, (3.1) (hence 3) holds if

$$
\sum_{k>0} \frac{\tau_{\mu, 2,2}(k)}{\sqrt{k}}<\infty
$$

In addition, since $\tau_{\mu, 2,2}(k) \leq\left(\tau_{\mu, 1,1}(k)\right)^{1 / 2}$, it follows that $(3.2)$ holds as soon as

$$
\sum_{k>0} \frac{\sqrt{\tau_{\mu, 1,1}(k)}}{\sqrt{k}}<\infty
$$

Proof of Theorem 2. We first prove that any of the conditions of Theorem 2 implies the weak convergence of $\sqrt{n}\left(F_{n}-F\right)$ in $\mathbb{B}=\mathbb{L}^{p}(\mu)$. A random variable $Z$ is in $\mathbb{L}^{2}(\mathbb{B})$ if \|\|$Z\left\|_{p, \mu}\right\|_{2}<\infty$. Let $Z_{i}=\left\{t \rightarrow \mathbb{I}_{X_{i} \leq t}-F(t)\right\}$. Clearly,

$$
\begin{aligned}
\left\|Z_{i}\right\|_{p, \mu} \leq\left(\int_{]-\infty, 0[}\left(\mathbb{I}_{X_{i} \leq t}\right)^{p} \mu(\mathrm{d} t)+\int_{[0, \infty[1}\left(1-\mathbf{I}_{X_{i} \leq t}\right)^{p} \mu(\mathrm{d} t)\right)^{1 / p} & +\left(\int_{]-\infty, 0[}(F(t))^{p} \mu(\mathrm{d} t)+\int_{[0, \infty[}(1-F(t))^{p} \mu(\mathrm{d} t)\right)^{1 / p},
\end{aligned}
$$

so that $\left\|Z_{i}\right\|_{p, \mu} \leq\left|F_{\mu}\left(X_{i}\right)\right|^{1 / p}+\mathbb{E}\left(\left|F_{\mu}\left(X_{i}\right)\right|^{1 / p}\right)$ and \|\|$Z_{i}\left\|_{p, \mu}\right\|_{2} \leq 2\left\|Y_{p, \mu}\right\|_{2}$.

Case 1. From the non ergodic version of Woyczyński's result (1975) [18], we know that if an $\mathcal{M}_{0}$-measurable r.v. $M_{0}$ in $\mathbb{L}^{2}(\mathbb{B})$ is such that $\mathbb{E}\left(M_{0} \mid \mathcal{M}_{-1}\right)=0$, then $n^{-1 / 2}\left(M_{0} \circ T+\cdots+M_{0} \circ T^{n}\right)$ converges in distribution to a random variable $M$. Now if

$$
U_{0}=M_{0}+N_{0}-N_{0} \circ T \text { and } U_{i}=U_{0} \circ T^{i}
$$

with $N_{0}$ in $\mathbb{L}^{2}(\mathbb{B})$, we easily infer that $n^{-1 / 2}\left(U_{1}+\cdots+U_{n}\right)$ also converges in distribution to $M$. Assume that $\sum_{k>0}\|\| \mathbb{E}\left(U_{k} \mid \mathcal{M}_{0}\right)\left\|_{p, \mu}\right\|_{2}<\infty$. From a well known decomposition of Gordin (1969) [10], we have

$$
U_{0}=\sum_{i=0}^{\infty} \mathbb{E}\left(U_{i} \mid \mathcal{M}_{0}\right)-\mathbb{E}\left(U_{i} \mid \mathcal{M}_{-1}\right)+\sum_{i=0}^{\infty} \mathbb{E}\left(U_{i} \mid \mathcal{M}_{-1}\right)-\sum_{i=1}^{\infty} \mathbb{E}\left(U_{i} \mid \mathcal{M}_{0}\right),
$$

provided $U_{0}$ is $\mathcal{M}_{0}$-measurable. Hence, (3.3) holds with $M_{0}=\sum_{i=0}^{\infty} \mathbb{E}\left(U_{i} \mid \mathcal{M}_{0}\right)-\mathbb{E}\left(U_{i} \mid \mathcal{M}_{-1}\right)$ and the coboundary $N_{0}=\sum_{i=0}^{\infty} \mathbb{E}\left(U_{i} \mid \mathcal{M}_{-1}\right)$. Applying the preceding remarks to the random variable $U_{i}=Z_{i}$, we infer that $\sqrt{n}\left(F_{n}-F\right)=n^{-1 / 2}\left(Z_{1}+\cdots+Z_{n}\right)$ converges in distribution in $\mathbb{B}$ as soon as $\sum_{k \geq 0}\|\| \mathbb{E}\left(Z_{k} \mid \mathcal{M}_{0}\right)\left\|_{p, \mu}\right\|_{2}$ is finite. To conclude, note that \|\| $\mathbb{E}\left(Z_{k} \mid \mathcal{M}_{0}\right)\left\|_{p, \mu}\right\|_{2}=\tau_{\mu, p, 2}(k)$. 
Case 2. The fact that $\sqrt{n}\left(F_{n}-F\right)$ converges in distribution in $\mathbb{B}$ follows from Corollary $2(\delta)$ in Dedecker and Merlevède (2003) [5], by noting that $\left\|Z_{0}\right\|_{2, \mu} \leq 2 \mu(\mathbb{R})^{1 / 2}$ and that \|\| $\mathbb{E}\left(Z_{k} \mid \mathcal{M}_{0}\right)\left\|_{2, \mu}\right\|_{1}=\tau_{\mu, 2,1}(k)$.

Case 3. The fact that $\sqrt{n}\left(F_{n}-F\right)$ converges in distribution in $\mathbb{B}$ follows from Corollary 3 in Dedecker and Merlevède (2003) [5] (the condition $F_{X_{0} \mid \mathcal{M}_{-\infty}}=F$ means that $\mathbb{E}\left(Z_{0} \mid \mathcal{M}_{-\infty}\right)=0$ a.s.).

The operator $\Gamma$. It remains to identify $\Gamma$. Let $\left(f_{i}\right)_{1 \leq i \leq k}$ be some functions of $W_{p^{\prime}, 1}(\mu)$ and $\left(\alpha_{i}\right)_{1 \leq i \leq k}$ some real numbers. We shall prove that $G_{n}\left(\alpha_{1} f_{1}+\cdots+\alpha_{k} f_{k}\right)$ converges to a random variable whose conditional distribution with respect to $\mathcal{I}$ is that of a Gaussian random variable with variance $\sum_{i=1}^{n} \sum_{j=1}^{n} \alpha_{i} \alpha_{j} \Gamma\left(f_{i}, f_{j}\right)$.

Let $h=\alpha_{1} f_{1}+\cdots+\alpha_{k} f_{k}$. In case 1 , note that, by Lemma 1

$$
\left\|\mathbb{E}\left(h\left(X_{k}\right) \mid \mathcal{M}_{0}\right)-\mathbb{E}\left(h\left(X_{k}\right)\right)\right\|_{2} \leq\left(\sum_{i=1}^{n}\left|\alpha_{i}\right|\right)\|\| \mathbb{E}\left(Z_{k} \mid \mathcal{M}_{0}\right)\left\|_{p, \mu}\right\|_{2}
$$

and the result follows from Theorem 1 in Dedecker and Rio (2000) [8].

In case 2 , note that

$$
\left\|\left(h\left(X_{0}\right)-\mathbb{E}\left(h\left(X_{0}\right)\right)\right) \mathbb{E}\left(h\left(X_{k}\right)-\mathbb{E}\left(h\left(X_{k}\right)\right) \mid \mathcal{M}_{0}\right)\right\|_{1} \leq\left(\sum_{i=1}^{n}\left|\alpha_{i}\right|\right)^{2} \mathbb{E}\left(\left\|Z_{0}\right\|_{2, \mu}\left\|\mathbb{E}\left(Z_{k} \mid \mathcal{M}_{0}\right)\right\|_{2, \mu}\right) .
$$

Since $\left\|Z_{0}\right\|_{2, \mu} \leq 2 \mu(\mathbb{R})^{1 / 2}$ and \|\| $\mathbb{E}\left(Z_{k} \mid \mathcal{M}_{0}\right)\left\|_{2, \mu}\right\|_{1}=\tau_{\mu, 2,1}(k)$, we infer that

$$
\sum_{k \geq 1}\left\|\left(h\left(X_{0}\right)-\mathbb{E}\left(h\left(X_{0}\right)\right)\right) \mathbb{E}\left(h\left(X_{k}\right)-\mathbb{E}\left(h\left(X_{k}\right)\right) \mid \mathcal{M}_{0}\right)\right\|_{1}<\infty,
$$

and the result follows from Theorem 1 in Dedecker and Rio (2000) [8].

In case 3, the result follows from (3.5) by applying Corollary 3 and Remark 6 in Dedecker and Merlevède (2003) [5] with $\mathbb{H}=\mathbb{R}$ (see Rem. 5 of the same paper for the expression of the covariance).

\section{Comparison of COEFficients AND EXAmples}

\subsection{Some upper bounds}

Let $\lambda$ be the Lebesgue measure on $\mathbb{R}$. The four coefficients

$$
\begin{aligned}
\tau(\mathcal{M}, X)=\tau_{\lambda, 1,1}(\mathcal{M}, X) & \varphi(\mathcal{M}, X)=\tau_{\lambda, 1, \infty}(\mathcal{M}, X) \\
\beta(\mathcal{M}, X)=\tau_{\lambda, \infty, 1}(\mathcal{M}, X) & \phi(\mathcal{M}, X)=\tau_{\lambda, \infty, \infty}(\mathcal{M}, X)
\end{aligned}
$$

have been introduced and studied in Dedecker and Prieur (2004) [6] and (2005) [7]. The authors have shown that these coefficents can be easily computed in many situations. The coefficients $\beta(\mathcal{M}, X)$ and $\phi(\mathcal{M}, X)$ are weaker than the usual $\beta$-mixing and $\phi$-mixing coefficients, and they can be computed also for many non mixing models. The following lemma shows how to compare $\tau_{\mu, p, q}(\mathcal{M}, X)$ with $\tau(\mathcal{M}, X), \varphi(\mathcal{M}, X), \beta(\mathcal{M}, X)$ and $\phi(\mathcal{M}, X)$.

Lemma 4. Let $X$ be a real-valued random variable and $\mathcal{M}$ a $\sigma$-algebra of $\mathcal{A}$.

1. For any $p, q$ in $[1, \infty]$ and any finite measure $\mu, \tau_{\mu, p, q}(\mathcal{M}, X) \leq \mu(\mathbb{R})^{1 / p} \phi(\mathcal{M}, X)$.

2. For any $p, q$ in $[1, \infty]$ and any finite measure $\mu, \tau_{\mu, p, q}(\mathcal{M}, X) \leq \mu(\mathbb{R})^{1 / p} \beta(\mathcal{M}, X)^{1 / q}$.

3. If $t \rightarrow \mu(]-\infty, t])$ is $K$-lipschitz, then for any $p, q$ in $[1, \infty], \tau_{\mu, p, q}(\mathcal{M}, X) \leq(K \varphi(\mathcal{M}, X))^{1 / p}$.

4. If $t \rightarrow \mu(]-\infty, t])$ is $K$-lipschitz, then for any $p$ in $[1, \infty]$ and $q \leq p, \tau_{\mu, p, q}(\mathcal{M}, X) \leq(K \tau(\mathcal{M}, X))^{1 / p}$.

Remark 6. Let $\left(X_{i}\right)_{i \in \mathbb{Z}}$ and $\mathcal{M}_{0}$ be as in Section 3, and let $\mu$ be some finite measure. From Lemma 4 and Theorem 2, we infer that $\sqrt{n}\left(F_{n}-F\right)$ converges in distribution in $\mathbb{L}^{p}(\mu)$ as soon as

1. $p$ belongs to $\left[2, \infty\left[\right.\right.$ and $\sum_{k>0} \phi\left(\mathcal{M}_{0}, X_{k}\right)<\infty$; 
2. $p$ belongs to $\left[2, \infty\left[\right.\right.$ and $\sum_{k>0} \sqrt{\beta\left(\mathcal{M}_{0}, X_{k}\right)}<\infty$;

3. $p=2$ and $\sum_{k>0} \beta\left(\mathcal{M}_{0}, X_{k}\right)<\infty$;

4. $p=2$ and $\sum_{k>0} \frac{\phi\left(\mathcal{M}_{0}, X_{k}\right)}{\sqrt{k}}<\infty$.

Arguing as in Dedecker and Merlevède (2003, p. 250) [5], one can prove that

$$
\tau_{\mu, 2,1}(k) \leq 18 \mu(\mathbb{R}) \alpha\left(\mathcal{M}_{0}, \sigma\left(X_{k}\right)\right),
$$

where $\alpha(\mathcal{A}, \mathcal{B})$ is the strong mixing coefficient of Rosenblatt between two $\sigma$-algebras $\mathcal{A}$ and $\mathcal{B}$. Hence, we obtain from the condition 2 of Theorem 2 that $\sqrt{n}\left(F_{n}-F\right)$ converges in distribution in $\mathbb{L}^{2}(\mu)$ as soon as $\sum_{k>0} \alpha\left(\mathcal{M}_{0}, \sigma\left(X_{k}\right)\right)<\infty$. Note that, when $\mu=\lambda_{1}$ where $\lambda_{1}$ is the Lebesgue measure on [0,1], Oliveira and Suquet (1995) [13] obtained the same result under the slightly stronger condition $\sum_{k>0} \alpha\left(\mathcal{M}_{0}, \sigma\left(X_{i}, i \geq k\right)\right)<\infty$ (this condition implies that the sequence $\left(X_{i}\right)_{i \in \mathbb{Z}}$ is ergodic, so that the limiting process is Gaussian). In Oliveira and Suquet (1998) [14], they proved the convergence of $\sqrt{n}\left(F_{n}-F\right)$ in $\mathbb{L}^{p}\left(\lambda_{1}\right)$ for $p>2$ under the condition $\alpha\left(\mathcal{M}_{0}, \sigma\left(X_{i}, i \geq k\right)\right)=O\left(n^{-p / 2-\epsilon}\right)$ for some positive $\epsilon$ (in both papers, the authors also consider the case of associated random variables). In this case, since $\tau_{\lambda_{1}, p, 2}(k) \leq\left(\tau_{\lambda_{1}, 2,1}(k)\right)^{2 / p}$, we infer from the condition 1 of Theorem 2 and (4.1), that the convergence in $\mathbb{L}^{p}\left(\lambda_{1}\right)$ for $p>2$ holds as soon as $\sum_{k>0}\left(\alpha\left(\mathcal{M}_{0}, \sigma\left(X_{k}\right)\right)\right)^{2 / p}<\infty$ which improves on the condition obtained in Oliveira and Suquet. Of course, if $\mu=\lambda_{1}$, one can also apply the result of Doukhan et al. (1995) [9]: since for $1<p^{\prime} \leq \infty$ the $\varepsilon$-entropy of the class $W_{p^{\prime}, 1}^{0}\left(\lambda_{1}\right)$ with respect to the $\mathbb{L}^{\infty}$-norm is of order $\varepsilon^{-1}$, it follows that $\sqrt{n}\left(F_{n}-F\right)$ converges in distribution in $\mathbb{L}^{p}\left(\lambda_{1}\right)$ for any $1 \leq p<\infty$ as soon as $\sum_{k>0} \beta\left(\mathcal{M}_{0}, \sigma\left(X_{i}, i \geq k\right)\right)<\infty$, where $\beta(\mathcal{A}, \mathcal{B})$ is the $\beta$-mixing coefficient of Rozanov and Volkonskii between two $\sigma$-algebras $\mathcal{A}$ and $\mathcal{B}$ (recall that $2 \alpha(\mathcal{A}, \mathcal{B}) \leq \beta(\mathcal{A}, \mathcal{B})$ ).

Proof of Lemma 4. Item 1 is clear. Item 2 follows from the inequality

$$
\|\| F_{X \mid \mathcal{M}}-F_{X}\left\|_{p, \mu}\right\|_{q} \leq \mu(\mathbb{R})^{1 / p}\left\|\sup _{t \in \mathbb{R}}\left|F_{X \mid \mathcal{M}}(t)-F_{X}(t)\right|\right\|_{1}^{1 / q} .
$$

To prove the items 3 and 4 , note that, if $t \rightarrow \mu(]-\infty, t])$ is $K$-lipschitz,

$$
\|\| F_{X \mid \mathcal{M}}-F_{X}\left\|_{p, \mu}\right\|_{q} \leq\left(\mathbb{E}\left(\left(\int\left|F_{X \mid \mathcal{M}}(t)-F_{X}(t)\right|^{p} K \mathrm{~d} t\right)^{q / p}\right)\right)^{1 / q} .
$$

For $q=\infty$, we obtain

$$
\|\| F_{X \mid \mathcal{M}}-F_{X}\left\|_{p, \mu}\right\|_{\infty} \leq\left(K\left\|\int\left|F_{X \mid \mathcal{M}}(t)-F_{X}(t)\right|^{p} \mathrm{~d} t\right\|_{\infty}\right)^{1 / p} \leq(K \varphi(\mathcal{M}, X))^{1 / p} .
$$

If $q \leq p$, point 4 follows from Jensen's inequality and Fubini:

$$
\|\| F_{X \mid \mathcal{M}}-F_{X}\left\|_{p, \mu}\right\|_{q} \leq\left(\int \mathbb{E}\left(\left|F_{X \mid \mathcal{M}}(t)-F_{X}(t)\right|^{p}\right) K \mathrm{~d} t\right)^{1 / p} \leq\left(K \int\left\|F_{X \mid \mathcal{M}}(t)-F_{X}(t)\right\|_{1} \mathrm{~d} t\right)^{1 / p} .
$$

Before giving detailed examples, we state the following useful bounds for $\left.\tau_{\mu, p, q}(\mathcal{M}, X)\right)$ when $\mu$ is a probability measure and $X$ has a continuous distribution function $F$.

Lemma 5. Let $(\Omega, \mathcal{A}, \mathbb{P})$ be a probability space, $X$ a real-valued random variable and $\mathcal{M}$ a $\sigma$-algebra of $\mathcal{A}$. Assume that $X$ has a continuous distribution function $F$. If $X^{*}$ is a random variable distributed as $X$ and independent of $\mathcal{M}$ then, for any $y \in[0,1]$, any probability measure $\mu$ and any $p, q$ in $[1, \infty]$, we have that

$$
\tau_{\mu, p, q}(\mathcal{M}, X) \leq y+\left\|\mathbb{E}\left(\mathbb{I}_{\left|F(X)-F\left(X^{*}\right)\right|>y} \mid \mathcal{M}\right)\right\|_{q}
$$


In particular, taking $y=\left\|F(X)-F\left(X^{*}\right)\right\|_{\infty}$ in the previous inequality, we obtain that

$$
\left.\tau_{\mu, p, q}(\mathcal{M}, X)\right) \leq\left\|F(X)-F\left(X^{*}\right)\right\|_{\infty}
$$

Now if $w$ is the modulus of continuity of $F$, then, for any any $x>0$ and any $r<\infty$ such that $r q \in[1, \infty]$,

$$
\left.\left.\tau_{\mu, p, q}(\mathcal{M}, X)\right) \leq w(x)+\left(\frac{\left\|X-X^{*}\right\|_{q r}}{x}\right)^{r} \quad \text { and } \quad \tau_{\mu, p, q}(\mathcal{M}, X)\right) \leq w\left(\left\|X-X^{*}\right\|_{\infty}\right)
$$

In particular, if $X$ has a density bounded by $K$, we have that

$$
\left.\left.\tau_{\mu, p, q}(\mathcal{M}, X)\right) \leq C(r)\left(K\left\|X-X^{*}\right\|_{q r}\right)^{r /(r+1)} \quad \text { and } \quad \tau_{\mu, p, q}(\mathcal{M}, X)\right) \leq K\left\|X-X^{*}\right\|_{\infty}
$$

with $C(r)=r^{1 /(r+1)}+r^{-r /(r+1)}($ note that $C(r) \leq 2$ and $C(\infty)=1)$.

Proof of Lemma 5. Note first that, since $\mu$ is a probability measure,

$$
\left.\tau_{\mu, p, q}(\mathcal{M}, X)\right) \leq\left\|\sup _{t \in \mathbb{R}} \mid F_{X \mid \mathcal{M}}(t)-F(t)\right\|_{q}
$$

Let $Y=F(X)$ and $Y^{*}=F\left(X^{*}\right)$. According to Lemma 3 in Dedecker and Prieur (2005) [7], we have that

$$
\sup _{t \in \mathbb{R}}\left|F_{X \mid \mathcal{M}}(t)-F(t)\right|=\sup _{t \in \mathbb{R}}\left|F_{Y \mid \mathcal{M}}(t)-F_{Y}(t)\right| .
$$

Since $F$ is continuous, $Y$ is uniformly distributed over [0,1]. From inequality (3.3) in Dedecker and Prieur (2005) [7], we obtain for any $y \in[0,1]$,

$$
\left|F_{Y \mid \mathcal{M}}(t)-t\right| \leq y+\mathbb{E}\left(\mathbb{I}_{\left|Y-Y^{*}\right|>y} \mid \mathcal{M}\right)
$$

and (4.2) follows. Now, since $\left|F(X)-F\left(X^{*}\right)\right| \leq w\left(\left|X-X^{*}\right|\right)$ we obtain that

$$
\left.\tau_{\mu, p, q}(\mathcal{M}, X)\right) \leq w(x)+\left\|\mathbb{E}\left(\mathbb{I}_{w\left(\left|F(X)-F\left(X^{*}\right)\right|\right)>w(x)} \mid \mathcal{M}\right)\right\|_{q} \leq w(x)+\left\|\mathbb{E}\left(\mathbf{I}_{\left|X-X^{*}\right|>x} \mid \mathcal{M}\right)\right\|_{q} .
$$

Applying Markov inequality at order $r$, we obtain (4.3). Finally, we prove (4.4) by noting that $w(x) \leq K x$ and by minimizing in $x$.

\subsection{Example 1: causal functions of stationary sequences}

Let $\left(\xi_{i}\right)_{i \in \mathbb{Z}}$ be a stationary sequence of random variables with values in a measurable space $\mathcal{X}$. Assume that there exists a function $H$ defined on a subset of $\mathcal{X}^{\mathbb{N}}$, with values in $\mathbb{R}$ and such that $H\left(\xi_{0}, \xi_{-1}, \xi_{-2}, \ldots,\right)$ is defined almost surely. The stationary sequence $\left(X_{n}\right)_{n \in \mathbb{Z}}$ defined by $X_{n}=H\left(\xi_{n}, \xi_{n-1}, \xi_{n-2}, \ldots\right)$ is called a causal function of $\left(\xi_{i}\right)_{i \in \mathbb{Z}}$.

Assume that there exists a stationary sequence $\left(\xi_{i}^{\prime}\right)_{i \in \mathbb{Z}}$ distributed as $\left(\xi_{i}\right)_{i \in \mathbb{Z}}$ and independent of $\left(\xi_{i}\right)_{i \leq 0}$. Define $X_{n}^{*}=H\left(\xi_{n}^{\prime}, \xi_{n-1}^{\prime}, \xi_{n-2}^{\prime}, \ldots\right)$. Clearly $X_{n}^{*}$ is independent of $\mathcal{M}_{0}=\sigma\left(\xi_{i}, i \leq 0\right)$ and distributed as $X_{n}$. For any $p \geq 1$ ( $p$ may be infinite) define the sequence $\left(\delta_{i, p}\right)_{i>0}$ by

$$
\left\|X_{i}-X_{i}^{*}\right\|_{p}=\delta_{i, p}
$$

From Lemma 5, we infer that, if $\mu$ is a probability measure and $X_{0}$ has a density bounded by $K$, then for any $p, q$ in $[1, \infty]$ and any $r$ such that $r q \in[1, \infty]$,

$$
\tau_{\mu, p, q}\left(\mathcal{M}_{0}, X_{k}\right) \leq C(r)\left(K \delta_{k, q r}\right)^{r /(r+1)} \quad \text { and } \quad \tau_{\mu, p, q}\left(\mathcal{M}_{0}, X_{k}\right) \leq K \delta_{k, \infty}
$$


In particular, these results apply to the case where the sequence $\left(\xi_{i}\right)_{i \in \mathbb{Z}}$ is $\beta$-mixing in the sense of Rozanov and Volkonskii. Let $\widetilde{\xi}_{k}=\left(\xi_{k}, \xi_{k+1}, \ldots\right), \mathcal{F}_{0}=\sigma\left(\xi_{i}, i \leq 0\right)$ and $\mathcal{G}_{k}=\sigma\left(\xi_{i}, i \geq k\right)$. Let $\mathbb{P}_{\widetilde{\xi}_{k}}$ be the distribution of $\widetilde{\xi}_{k}$, and let $\mathbb{P}_{\widetilde{\xi}_{k} \mid \mathcal{F}_{0}}$ be a conditional distribution of $\widetilde{\xi}_{k}$ given $\mathcal{F}_{0}$. According to Theorem 4.4 .7 in Berbee (1979) [2], if $\Omega$ is rich enough, there exists $\left(\xi_{i}^{\prime}\right)_{i \in \mathbb{Z}}$ distributed as $\left(\xi_{i}\right)_{i \in \mathbb{Z}}$ and independent of $\left(\xi_{i}\right)_{i \leq 0}$ such that

$$
\mathbb{P}\left(\xi_{i} \neq \xi_{i}^{\prime} \text { for some } i \geq k\right)=\frac{1}{2} \mathbb{E}\left(\left\|\mathbb{P}_{\widetilde{\xi}_{k} \mid \mathcal{F}_{0}}-\mathbb{P}_{\widetilde{\xi}_{k}}\right\|_{v}\right)=\beta\left(\mathcal{F}_{0}, \mathcal{G}_{k}\right)
$$

where $\|\cdot\|_{v}$ is the variation norm. If the sequence $\left(\xi_{i}\right)_{i \in \mathbb{Z}}$ is iid, it suffices to take $\xi_{i}^{\prime}=\xi_{i}$ for $i>0$ and $\xi_{i}^{\prime}=\xi_{i}^{\prime \prime}$ for $i \leq 0$, where $\left(\xi_{i}^{\prime \prime}\right)_{i \in \mathbb{Z}}$ is an independent copy of $\left(\xi_{i}\right)_{i \in \mathbb{Z}}$.

Application: causal linear processes. In that case $X_{n}=\sum_{j \geq 0} a_{j} \xi_{n-j}$. For any $p \geq 1$, we have that

$$
\delta_{i, p} \leq \sum_{j \geq 0}\left|a_{j}\right|\left\|\xi_{i-j}-\xi_{i-j}^{\prime}\right\|_{p} \leq\left\|\xi_{0}-\xi_{0}^{\prime}\right\|_{p} \sum_{j \geq i}\left|a_{j}\right|+\sum_{j=0}^{i-1}\left|a_{j}\right|\left\|\xi_{i-j}-\xi_{i-j}^{\prime}\right\|_{p} .
$$

From Proposition 2.3 in Merlevède and Peligrad (2002) [12], we obtain that

$$
\delta_{i, p} \leq\left\|\xi_{0}-\xi_{0}^{\prime}\right\|_{p} \sum_{j \geq i}\left|a_{j}\right|+\sum_{j=0}^{i-1}\left|a_{j}\right|\left(2^{p} \int_{0}^{\beta\left(\mathcal{F}_{0}, \mathcal{G}_{i-j}\right)} Q_{\xi_{0}}^{p}(u)\right)^{1 / p} \mathrm{~d} u .
$$

where $Q_{\xi_{0}}$ is the cadlag inverse of the tail function $x \rightarrow \mathbb{P}\left(\left|\xi_{0}\right|>x\right)$.

If the sequence $\left(\xi_{i}\right)_{i \in \mathbb{Z}}$ is iid, it follows that $\delta_{i, p} \leq\left\|\xi_{0}-\xi_{0}^{\prime}\right\|_{p} \sum_{j \geq i}\left|a_{j}\right|$. Moreover, for $p=2$ we have exactly $\delta_{i, 2}=\left(2 \operatorname{Var}\left(\xi_{0}\right) \sum_{j \geq i} a_{j}^{2}\right)^{1 / 2}$. For instance, if $a_{i}=2^{-i-1}$ and $\xi_{0} \sim \mathcal{B}(1 / 2)$, then $\delta_{i, \infty} \leq 2^{-i}$. Since $X_{0}$ is uniformly distributed over $[0,1]$, we have $\tau_{\mu, p, q}\left(\mathcal{M}_{0}, X_{i}\right) \leq 2^{-i}$. Recall that this sequence is not strongly mixing in the sense of Rosenblatt.

\subsection{Example 2: iterated random functions}

Let $\left(X_{n}\right)_{n>0}$ be a real-valued stationary Markov chain, such that $X_{n}=F\left(X_{n-1}, \xi_{n}\right)$ for some measurable function $F$ and some iid sequence $\left(\xi_{i}\right)_{i>0}$ independent of $X_{0}$. Let $X_{0}^{*}$ be a random variable distributed as $X_{0}$ and independent of $\left(X_{0},\left(\xi_{i}\right)_{i>0}\right)$. Define $X_{n}^{*}=F\left(X_{n-1}^{*}, \xi_{n}\right)$. The sequence $\left(X_{n}^{*}\right)_{n \geq 0}$ is distributed as $\left(X_{n}\right)_{n \geq 0}$ and independent of $X_{0}$. Let $\mathcal{M}_{i}=\sigma\left(X_{j}, 0 \leq j \leq i\right)$. As in Example 1, define the sequence $\left(\delta_{i, p}\right)_{i>0}$ by $(4.5)$. From Lemma 5 , we infer that, if $\mu$ is a probability measure and $X_{0}$ has a density bounded by $K$, then for any $p, q$ in $[1, \infty]$ and any $r$ such that $r q \in[1, \infty]$, the bounds given in (4.6) hold.

Let $\nu$ be the distribution of $X_{0}$ and $\left(X_{n}^{x}\right)_{n \geq 0}$ the chain starting from $X_{0}^{x}=x$. With these notations, we have

$$
\delta_{i, p}^{p}=\iint\left\|X_{i}^{x}-X_{i}^{y}\right\|_{p}^{p} \mu(\mathrm{d} x) \mu(\mathrm{d} y) .
$$

For instance, if there exists a sequence $\left(d_{i, p}\right)_{i \geq 0}$ of positive numbers such that

$$
\left\|X_{i}^{x}-X_{i}^{y}\right\|_{p} \leq d_{i, p}|x-y|
$$

then $\delta_{i, p} \leq d_{i, p}\left\|X_{0}-X_{0}^{*}\right\|_{p}$. In the usual case where $\left\|F\left(x, \xi_{0}\right)-F\left(y, \xi_{0}\right)\right\|_{p} \leq \kappa|x-y|$ for some $\kappa<1$, we can take $d_{i, p}=\kappa^{i}$.

An important example is $X_{n}=f\left(X_{n-1}\right)+\xi_{n}$ for some $\kappa$-lipschitz function $f$. If $X_{0}$ has a moment of order $p$, then $\delta_{i, p} \leq \kappa^{i}\left\|X_{0}-X_{0}^{*}\right\|_{p}$. In particular, if $X_{0}$ is bounded and has a density bounded by $K$ then $\tau_{\mu, p, q}\left(\mathcal{M}_{0}, X_{i}\right) \leq 2 K\left\|X_{0}\right\|_{\infty} \kappa^{i}$. 


\section{Applications}

\subsection{Iterates of expanding maps}

Let $I=[0,1], T$ be a map from $I$ to $I$ and define $X_{i}=T^{i}$. If the probability $\pi$ is invariant by $T$, the sequence $\left(X_{i}\right)_{i \geq 0}$ of random variables from $(I, \pi)$ to $I$ is strictly stationary. Denote by $\|g\|_{1, \lambda_{1}}$ the $\mathbb{L}^{1}$-norm with respect to the Lebesgue measure $\lambda_{1}$ on $[0,1]$ and by $\|\nu\|=|\nu|(I)$ the total variation of a signed measure $\nu$. Let $\mathcal{M}_{n}=\sigma\left(X_{i}, i \geq n\right)$. In many interesting cases (see Sect. 4.4 in Dedecker and Prieur (2005) [7]), one can prove that, for any bounded variation function $h$ on $I$ and any integrable $\mathcal{M}_{n}$-measurable random variable $Y$,

$$
\left|\operatorname{Cov}\left(h\left(X_{0}\right), Y\right)\right| \leq a_{n}\|Y\|_{1}\left(\|h\|_{1, \lambda_{1}}+\|\mathrm{d} h\|\right),
$$

for some nonincreasing sequence $a_{n}$ tending to zero as $n$ tends to infinity. Note that if (5.1) holds, then $\left|\operatorname{Cov}\left(h\left(X_{0}\right), Y\right)\right|=\left|\operatorname{Cov}\left(h\left(X_{0}\right)-h(0), Y\right)\right| \leq a_{n}\|Y\|_{1}\left(\|h-h(0)\|_{1, \lambda_{1}}+\|\mathrm{d} h\|\right)$. Since $\|h-h(0)\|_{1, \lambda_{1}} \leq\|\mathrm{d} h\|$, we obtain that

$$
\left|\operatorname{Cov}\left(h\left(X_{0}\right), Y\right)\right| \leq 2 a_{n}\|Y\|_{1}\|\mathrm{~d} h\| .
$$

From Lemma 4 in Dedecker and Prieur (2005) [7], we infer that if the inequality (5.2) holds, then $\phi\left(\mathcal{M}_{n}, X_{0}\right) \leq$ $2 a_{n}$. Applying Lemma 4 , we infer that $\tau_{\lambda_{1}, p, \infty}\left(\mathcal{M}_{n}, X_{0}\right) \leq 2 a_{n}$ for any $p$ in $[1, \infty]$. In particular, it follows from (2.8) that

$$
\mathbb{P}\left(\sqrt{n} K\left(F_{n}, F\right) \geq x\right) \leq \mathbb{P}\left(\sqrt{n} D_{2, n}\left(\lambda_{1}\right) \geq x\right) \leq 2 \exp \left(-\frac{x^{2}}{2\left(1+4\left(a_{1}+\cdots+a_{n-1}\right)\right)^{2}}\right) .
$$

In a recent paper, Collet, Martinez and Schmitt (2002) [4] studied a class of expanding maps for which (5.1) (and hence (5.2)) holds with $a_{n}=C \rho^{n}$ for some $\rho<1$. Using a concentration inequality for Lipschitz functions they prove in Theorem III.1 that there exist a number $x_{0}>0$ and a constant $R>0$ (both depending on $T$ ) such that, for any $x>x_{0}$ and any integer $n$,

$$
\mathbb{P}\left(\sqrt{n} K\left(F_{n}, F\right) \geq x\right) \leq \exp \left(-R x^{2}\right) .
$$

Clearly, (5.3) is more precise than (5.4), for it holds for any positive $x$. Moreover, we do not require that $a_{n}$ decreases geometrically, and we are able to give an expression for $R$ in terms of the coefficients $\left(a_{i}\right)_{1 \leq i \leq n-1}$.

\subsection{Causal linear processes}

The main interest of the coefficients $\tau_{\mu, p, q}$ is that they are very easy to evaluate in many situations. Let us focus on the stationary sequence

$$
X_{k}=\sum_{j \geq 0} a_{j} \xi_{k-j}
$$

where $\left(\xi_{i}\right)_{i \in \mathbb{Z}}$ is a sequence of iid random variables and $\sum_{j>0}\left|a_{j}\right|<\infty$. From (4.6) and the application of Section 4.2 , we know that if $\mu$ is a probability measure and if $\bar{X}_{0}$ has a density bounded by $K$, then for any $p, q$ in $[1, \infty]$ and any $r$ such that $r q \in[1, \infty]$,

$$
\tau_{\mu, p, q}\left(\mathcal{M}_{0}, X_{n}\right) \leq C(r)\left(K\left\|\xi_{0}-\xi_{1}\right\|_{r q} \sum_{j \geq n}\left|a_{j}\right|\right)^{r /(r+1)} \quad \text { and } \quad \tau_{\mu, p, \infty}\left(\mathcal{M}_{0}, X_{n}\right) \leq K\left\|\xi_{0}-\xi_{1}\right\|_{\infty} \sum_{j \geq n}\left|a_{j}\right|
$$

where $\mathcal{M}_{0}=\sigma\left(\xi_{k}, k \leq 0\right)$. For instance, if we use the last bound in (5.6), we obtain from (2.3) that

$$
\mathbb{P}\left(\sqrt{n} D_{p, n}(\mu) \geq x\right) \leq 2 \exp \left(-\frac{x^{2}}{2(p-1)\left(1+4 K\left\|\xi_{0}\right\|_{\infty} \sum_{j=1}^{\infty}(n \wedge j)\left|a_{j}\right|\right)^{2}}\right) .
$$


Applying this result in the particular case $\mu=\delta_{t}$, we infer that

$$
\sup _{t \in \mathbb{R}} \mathbb{P}\left(\sqrt{n}\left|F_{n}(t)-F(t)\right| \geq x\right) \leq 2 \exp \left(-\frac{x^{2}}{2\left(1+4 K\left\|\xi_{0}\right\|_{\infty} \sum_{j=1}^{\infty}(n \wedge j)\left|a_{j}\right|\right)^{2}}\right),
$$

which is similar (up to numerical constants) to the upper bound obtained in Corollary 2 in Rio (2000) [16]. In that case, the bound (5.8) can be improved by using the inequality (2.9).

From Condition 1 of Theorem 2, we infer that $\sqrt{n}\left(F_{n}-F\right)$ converges in distribution in any $\mathbb{L}^{p}(\mu)$, where $\mu$ is a probability measure and $2 \leq p<\infty$, as soon as $\left\|\xi_{0}\right\|_{2 r}<\infty$ and $\sum_{k>0}\left(\sum_{j \geq k}\left|a_{j}\right|\right)^{r /(r+1)}<\infty$ for some $r$ in $[1 / 2, \infty]$. From Condition $(3.2)$, we infer that $\sqrt{n}\left(F_{n}-F\right)$ converges in distribution in $\mathbb{L}^{2}(\mu)$ as soon as $\left\|\xi_{0}\right\|_{2 r}<\infty$ and $\sum_{k>0} k^{-1 / 2}\left(\sum_{j>k}\left|a_{j}\right|\right)^{r /(r+1)}<\infty$. In particular, the latter condition is realized for bounded innovations provided $\left|a_{i}\right|=O\left(i^{-3 / 2-\epsilon}\right)$ for some positive $\epsilon$.

Note that all the results mentioned above are valid without assuming that the innovations have a density : we have only assumed that $X_{0}$ has a density, which is much weaker (think of the well know example where $a_{i}=2^{-i-1}$ and $\left.\xi_{0} \sim \mathcal{B}(1 / 2)\right)$. Now, if we assume that $\xi_{0}$ has a density bounded by $C$, we can also evaluate the quantities \|\|$F_{X_{k} \mid \mathcal{M}_{i}}-F_{X_{k} \mid \mathcal{M}_{i-1}}\left\|_{p, \mu}\right\|_{q}$ which appear in the inequality (2.1) and in Condition 3 of Theorem 2.

Lemma 6. Let $\left(X_{k}\right)_{k>0}$ be defined by (5.5) and let $\mathcal{M}_{i}=\sigma\left(\xi_{k}, k \leq i\right)$. If $\xi_{0}$ has a density bounded by $C$, then, for any probability measure $\mu$ and any $p, q$ in $[1, \infty]$,

$$
\|\| F_{X_{k} \mid \mathcal{M}_{0}}-F_{X_{k} \mid \mathcal{M}_{-1}}\left\|_{p, \mu}\right\|_{q} \leq C\left\|\xi_{1}-\xi_{0}\right\|_{q} \frac{\left|a_{k}\right|}{\left|a_{0}\right|} .
$$

Applying Lemma 6, and arguing as for the proof of Inequality (2.1), we infer that,

$$
\mathbb{P}\left(\sqrt{n} D_{p, n}(\mu) \geq x\right) \leq 2 \exp \left(-\frac{x^{2}}{2(p-1)\left(1+2\left|a_{0}\right|^{-1} C\left\|\xi_{0}\right\|_{\infty} \sum_{k=1}^{\infty}\left|a_{k}\right|\right)^{2}}\right)
$$

which is more precise than (5.7), since it gives $\mathbb{P}\left(\sqrt{n} D_{p, n}(\mu) \geq x\right) \leq 2 \exp \left(-R x^{2}\right)$ for some positive constant $R$ as soon as $\sum_{i>0}\left|a_{i}\right|<\infty$. In the same way, we infer from Condition 3 of Theorem 2 that $\sqrt{n}\left(F_{n}-F\right)$ converges in distribution in $\mathbb{L}^{2}(\mu)$ as soon as $\left\|\xi_{0}\right\|_{2}<\infty$ and $\sum_{i>0}\left|a_{i}\right|<\infty$.

Proof of Lemma 6. Let $Y_{k}=\sum_{i=0}^{k-1} a_{i} \xi_{k-i}$. Clearly

$$
F_{X_{k} \mid \mathcal{M}_{0}}(t)=F_{Y_{k}}\left(t-\left(X_{k}-Y_{k}\right)\right) \quad \text { and } \quad F_{X_{k} \mid \mathcal{M}_{-1}}(t)=\int F_{Y_{k}}\left(t-a_{k} x-\left(X_{k}-Y_{k+1}\right)\right) P_{\xi_{0}}(\mathrm{~d} x) .
$$

Let $f_{\xi}$ be the density of $\xi_{0}$. The density of $Y_{k}$ is given by $f_{Y_{k}}=\left|a_{0}\right|^{-1} f_{\xi}\left(\cdot / a_{0}\right) * \cdots *\left|a_{k-1}\right|^{-1} f_{\xi}\left(\cdot / a_{k-1}\right)$, and hence it is bounded by $C\left|a_{0}\right|^{-1}$. Consequently

$$
\left|F_{X_{k} \mid \mathcal{M}_{0}}(t)-F_{X_{k} \mid \mathcal{M}_{-1}}(t)\right| \leq C\left|a_{0}\right|^{-1}\left|a_{k}\right| \int\left|x-\xi_{0}\right| P_{\xi_{0}}(\mathrm{~d} x)
$$

The result follows by taking the $\|\cdot\|_{q}$-norm and applying Jensen's inequality.

\section{Appendix: proof of Lemma 1.}

Let $f \in W_{p^{\prime}, 1}(\mu)$. We first check that under (1.1), $|f|$ is integrable with respect to $\mathrm{d} F$. Without loss of generality, assume that $f(0)=0$. Clearly

$$
\int|f(t)| \mathrm{d} F(t) \leq \int_{\mathbb{R}^{+}}\left(\int_{[0, t[}\left|f^{\prime}(x)\right| \mu(\mathrm{d} x)\right) \mathrm{d} F(t)+\int_{\mathbb{R}^{-}}\left(\int_{[t, 0[}\left|f^{\prime}(x)\right| \mu(\mathrm{d} x)\right) \mathrm{d} F(t) .
$$


Applying Fubini, we obtain that

$$
\int|f(t)| \mathrm{d} F(t) \leq \int_{\mathbb{R}^{+}}\left|f^{\prime}(x)\right|(1-F(x)) \mu(\mathrm{d} x)+\int_{\mathbb{R}^{-}}\left|f^{\prime}(x)\right| F(x) \mu(\mathrm{d} x) .
$$

Since $f^{\prime}$ belongs to $\mathbb{L}^{p^{\prime}}(\mu)$, the right hand side is finite as soon as (1.1) holds. In the same way, we have both

$$
\begin{aligned}
& \int f(t) \mathrm{d} F(t)=\int_{\mathbb{R}^{+}} f^{\prime}(x)(1-F(x)) \mu(\mathrm{d} x)-\int_{\mathbb{R}^{-}} f^{\prime}(x) F(x) \mu(\mathrm{d} x) \\
& \int f(t) \mathrm{d} G(t)=\int_{\mathbb{R}^{+}} f^{\prime}(x)(1-G(x)) \mu(\mathrm{d} x)-\int_{\mathbb{R}^{-}} f^{\prime}(x) G(x) \mu(\mathrm{d} x) .
\end{aligned}
$$

Consequently

$$
\int f(t) \mathrm{d} F(t)-\int f(t) \mathrm{d} G(t)=\int f^{\prime}(x)(G(x)-F(x)) \mu(\mathrm{d} x)
$$

The second equality follows by noting that

$$
\|F-G\|_{p, \mu}=\sup _{\|g\|_{p^{\prime}, \mu} \leq 1}\left|\int_{\mathbb{R}} g(x)(F(x)-G(x)) \mathrm{d} \mu(x)\right| .
$$

Acknowledgements. The authors are indebted to the referees for carefully reading the manuscript and for helpful comments.

\section{REFERENCES}

[1] K. Azuma, Weighted sums of certain dependent random variables. Tôkohu Math. J. 19 (1967) 357-367.

[2] H.C.P. Berbee, Random walks with stationary increments and renewal theory. Mathematical Centre Tracts 112, Mathematisch Centrum, Amsterdam (1979).

[3] L. Birgé and P. Massart, An adaptive compression algorithm in Besov Spaces. Constr. Approx. 16 (2000) 1-36.

[4] P. Collet, S. Martinez and B. Schmitt, Exponential inequalities for dynamical measures of expanding maps of the interval. Probab. Theory Relat. Fields 123 (2002) 301-322.

[5] J. Dedecker and F. Merlevède, The conditional central limit theorem in Hilbert spaces. Stoch. Processes Appl. 108 (2003) $229-262$.

[6] J. Dedecker and C. Prieur, Coupling for $\tau$-dependent sequences and applications. J. Theoret. Probab. 17 (2004) 861-885.

[7] J. Dedecker and C. Prieur, New dependence coefficients. Examples and applications to statistics. Probab. Theory Relat. Fields 132 (2005) 203-236.

[8] J. Dedecker and E. Rio, On the functional central limit theorem for stationary processes. Ann. Inst. H. Poincaré Probab. Statist. 36 (2000) 1-34.

[9] P. Doukhan, P. Massart and E. Rio, Invariance principle for absolutely regular empirical processes. Ann. Inst. H. Poincaré Probab. Statist. 31 (1995) 393-427.

[10] M.I. Gordin, The central limit theorem for stationary processes. Dokl. Akad. Nauk SSSR 188 (1969) 739-741.

[11] P. Massart, The tight constant in the Dvoretzky-Kiefer-Wolfowitz inequality. Ann. Probab. 18 (1990) 1269-1283.

[12] F. Merlevède and M. Peligrad, On the coupling of dependent random variables and applications, in Empirical process techniques for dependent data, Birkhäuser (2002) 171-193.

[13] P. Oliveira and C. Suquet, $\mathbb{L}^{2}([0,1])$ weak convergence of the empirical process for dependent variables, in Wavelets and statistics (Villard de Lans 1994), Lect. Notes Statist. 103 (1995) 331-344.

[14] P. Oliveira and C. Suquet, Weak convergence in $\mathbb{L}^{p}([0,1])$ of the uniform empirical process under dependence. Statist. Probab. Lett. 39 (1998) 363-370.

[15] I.F. Pinelis, An approach to inequalities for the distributions of infinite-dimensional martingales, in Probability in Banach spaces, Proc. Eight Internat. Conf. 8 (1992) 128-134.

[16] E. Rio, Inégalités de Hoeffding pour les fonctions lipschitziennes de suites dépendantes. C. R. Acad. Sci. Paris Série I 330 (2000) 905-908.

[17] A.W. van der Vaart, Bracketing smooth functions. Stoch. Processes Appl. 52 (1994) 93-105.

[18] W.A. Woyczyński, A central limit theorem for martingales in Banach spaces. Bull. Acad. Polon. Sci. Sr. Sci. Math. Astronom. Phys. 23 (1975) 917-920.

[19] V.V. Yurinskii, Exponential bounds for large deviations. Theory Prob. Appl. 19 (1974) 154-155. 